\title{
Social organization and the evolution of cumulative technology in apes and hominins
}

\author{
Pradhan, Gauri R ; Tennie, Claudio ; van Schaik, Carel P
}

\begin{abstract}
Culturally supported accumulation (or ratcheting) of technological complexity is widely seen as characterizing hominin technology relative to that of the extant great apes, and thus as representing a threshold in cultural evolution. To explain this divide, we modeled the process of cultural accumulation of technology, which we defined as adding new actions to existing ones to create new functional combinations, based on a model for great ape tool use. The model shows that intraspecific and interspecific variation in the presence of simple and cumulative technology among extant orangutans and chimpanzees is largely due to variation in sociability, and hence opportunities for social learning. The model also suggests that the adoption of extensive allomaternal care (cooperative breeding) in early Pleistocene Homo, which led to an increase in sociability and to teaching, and hence increased efficiency of social learning, was enough to facilitate technological ratcheting. Hence, socioecological changes, rather than advances in cognitive abilities, can account for the cumulative cultural changes seen until the origin of the Acheulean. The consequent increase in the reliance on technology could have served as the pacemaker for increased cognitive abilities. Our results also suggest that a more important watershed in cultural evolution was the rise of donated culture (technology or concepts), in which technology or concepts was transferred to naïve individuals, allowing them to skip many learning steps, and specialization arose, which allowed individuals to learn only a subset of the population's skills.
\end{abstract}

DOI: https://doi.org/10.1016/j.jhevol.2012.04.008

Posted at the Zurich Open Repository and Archive, University of Zurich

ZORA URL: https://doi.org/10.5167/uzh-71087

Journal Article

Accepted Version

Originally published at:

Pradhan, Gauri R; Tennie, Claudio; van Schaik, Carel P (2012). Social organization and the evolution of cumulative technology in apes and hominins. Journal of Human Evolution, 63(1):180-190.

DOI: https://doi.org/10.1016/j.jhevol.2012.04.008 


\section{SOCIAL ORGANIZATION AND THE EVOLUTION OF CUMULATIVE TECHNOLOGY}

2 IN APES AND HOMININS

3

4 Gauri R. Pradhan ${ }^{1,2}$

5 Claudio Tennie ${ }^{3}$

6 Carel van Schaik ${ }^{1}$

7

8 1: Anthropological Institute \& Museum, University of Zurich, Winterthurerstrasse 190,

$9 \mathrm{CH}-8057$ Zurich, Switzerland

10 2: Department of Physics, University of South Florida, Tampa, FL 33620, USA

11 3: Department of Developmental and Comparative Psychology, Max Planck Institute for

12 Evolutionary Anthropology, Deutscher Platz 6, 04103 Leipzig, Germany

13

14

15 Corresponding author:

16 Gauri R. Pradhan

174202 E Fowler Ave, ISA110

18 Department of Physics, University of South Florida, Tampa, FL 33620, USA

19 Tel: +1-(813) 9742872

20 Fax: +1-(813) 9745813

21 Email: gauri@usf.edu

22

23

24 


\begin{abstract}
1 Abstract
2 Culturally supported accumulation (or ratcheting) of technological complexity is widely

3 seen as characterizing hominin technology relative to that of the extant great apes, and

4 thus as representing a watershed in cultural evolution. To explain this divide, we

5 modeled the process of cultural accumulation of technology, which we defined as adding

6 new actions to existing ones to create new functional combinations, based on a model

7 for great ape tool use. The model shows that intraspecific and interspecific variation in

8 the presence of simple and cumulative technology among extant orangutans and

9 chimpanzees is largely due to variation in sociability, and hence opportunities for social

10 learning. The model also suggests that the adoption of extensive allomaternal care

11 (cooperative breeding) in early Pleistocene Homo, which led to an increase in sociability

12 and to teaching, and hence increased efficiency of social learning, was enough to

13 facilitate technological ratcheting. Hence, socioecological changes, rather than advances

14 in cognitive abilities, can account for the cumulative cultural changes seen until the origin

15 of the Acheulean. The consequent increase in the reliance on technology could have

16 served as the pacemaker for increased cognitive abilities. Our results also suggest that a

17 more important watershed in cultural evolution was the rise of donated culture
\end{abstract}

18 (technology or concepts), in which technology or concepts was transferred to naïve

19 individuals, allowing them to skip many learning steps, and specialization arose, which

20 allowed individuals to learn only a subset of the population's skills.

21

22 Key words: cumulative cultural evolution, ratcheting, hominin, great apes, sociability,

23 mathematical model, innovation, imitation

24

25 


\section{Introduction}

2 Recent studies have inferred the presence of culture, defined as multiple socially

3 transmitted innovations, in chimpanzees and orangutans, based on geographic variation

4 in behavior patterns or artifacts without obvious ecological or genetic correlates (Whiten

5 et al., 1999; van Schaik et al., 2003a; Boesch, 2003; Kruetzen et al. 2011) and indirect

6 indications of social learning in the field (Biro et al., 2003; Lonsdorf et al., 2004; Gruber

7 et al., 2009, Jaeggi et al., 2010; Reader and Biro, 2010). These studies have allowed us

8 to define more clearly what distinguishes human culture from that of the great apes,

9 whose cultures probably closely resemble those of the last common ancestor of humans

10 and the two chimpanzee species. Two major differences have emerged (Tomasello,

11 1999; van Schaik, 2004; Hill, 2009; Tennie et al., 2009): the cumulative nature of human

12 technology, and the cumulative and normative nature of human cultural institutions. Our

13 focus here is on explaining the origin of cumulative technology, which is widely

14 considered to represent a watershed in cultural evolution.

15 The prevailing explanation is that cumulative technology is absent in great apes

16 because they cannot imitate, and thus cannot reproduce novel actions with sufficient

17 precision to serve as a uniform foundation for subsequent addition of accumulations.

18 Thus, cumulative technology was thought to have arisen with Oldowan flake tools (Galef,

19 1992; Tomasello et al., 1993; Tomasello, 1994; Tomasello, 1999; Boyd and Richerson,

20 1996). We think this hypothesis is no longer supported, for two reasons. First, great apes

21 in experiments can reliably transmit complex techniques, although the exact

22 mechanisms remain debated, and second, they show some evidence of cumulative

23 technology, if properly defined.

24 With respect to the imitation question, despite much recent work on nonhuman

25 primates, no consensus on the mechanisms of observational social learning has

26 emerged (Byrne and Tanner, 2006; Tennie et al., 2009; Whiten et al., 2009).

27 Nonetheless, great apes have now been found to copy complex skills with sufficient

28 reliability to maintain basic behavioral uniformity in two-target experiments in captivity

29 despite the presence of alternative outcomes (reviews: Whiten et al., 2007; Whiten and

30 Mesoudi, 2008; Dindo et al., 2011), which would suffice to maintain systematic

31 differences in technology between nearby populations in the wild (Boesch, et al., 1994;

32 van Schaik and Knott, 2001). Although some doubt remains (Tennie et al., 2006;

33 Claidière and Sperber, 2010), most now agree that we should therefore look elsewhere 
1 than mechanisms of social learning to explain the elaboration of cumulative culture in

2 humans (Price et al., 2009; Tomasello 2009).

3 To evaluate possible evidence for cumulative technology among great apes, we

4 need a workable operational definition of cumulative technology, i.e. cumulative

5 technological innovations that have been transmitted socially to the point of having

6 reached high prevalence in a given population (habitual or customary status sensu

7 Whiten et al., 1999). Cumulative innovations have been defined as those beyond what a

8 naïve individual could invent during its lifetime (Galef, 1992; Tomasello, et al., 1993;

9 Boyd and Richerson, 1996), i.e. outside its Zone of Latent Solutions (Tennie et al.,

10 2009). However, this definition in effect assumes that the accumulation process has

11 already proceeded to the point that it has become impossible for naïve individuals to

12 invent the whole series of steps. It therefore excludes the initial steps of the

13 accumulation process, i.e. those that may still be invented by an individual, which

14 arguably are the very steps that historically determined the difference between

15 cumulative and non-cumulative culture.

16 We therefore adopted an alternative approach. The build-up "implies the

17 existence of superordinate representations abstracted from, and maintained over, the

18 course of multiple subordinate events" (Stout, 2011), and is therefore usually

19 accompanied by an increase in the size of the working memory, making the action series

20 cognitively more challenging as it gets longer (Price et al., 2009). We accordingly

21 defined the metric for the degree of accumulation (a.k.a. ratcheting) of a technique or

22 learned skill as the number of distinct actions integrated as steps in a single functional

23 sequence to reach an overall goal. One advantage of this metric is that this kind of

24 complexity corresponds closely to that in terms of techno-units Oswalt (1976), which

25 directly reflect properties of the tools themselves. It is in line with metrics developed for

26 primate food processing (Byrne, 1995; Matsuzawa, 1996), and is also very similar to

27 comparable metrics developed in archeology (Haidle, 2010; Stout, 2011), although we

28 did not admit other criteria, such as the complexity of each individual action (which is

29 also hard to define; Uomini, 2009) or the selectivity of the choice of raw material that is

30 used to produce the tool. We excluded them because these aspects can be gradually

31 improved over time through individual practice based on simple processes like

32 associative learning, once the basic action has been put in place by ratcheting (e.g.

33 Nonaka et al., 2010). It is important to stress that this system is preliminary and needs to 
1 be validated empirically through actual studies (for a recent attempt, see Sanz \&

2 Morgan, 2010). We will revisit this issue in the discussion.

3 The paradigmatic case of ratcheting is when an individual adds an existing

4 technique used in a very different context or an entirely novel one to an existing one, and

5 integrates them functionally. This can produce either a tool set (two or more tools used

6 consecutively in a functionally integrated way), a composite tool (two existing tools

7 combined directly), or a more complex tool (where subsequent actions modify an

8 existing tool, adding functionality to it). Table 1 provides the definitions of the first

9 ratcheting steps that can be recognized using this criterion, producing increasing

10 technology levels (TL), and provides examples for both stick and stone tools.

11 Although cumulative technology defined this way is absent among orangutans,

12 various examples have recently emerged for chimpanzees (Sanz et al., 2004; Sanz and

13 Morgan, 2007; Sanz et al., 2009; Boesch et al., 2009). In the Goualougo triangle, for

14 instance, the local chimpanzees use a tool set, consisting of a stout puncturing stick and

15 a slender probe, to exploit subterranean termite nests. It is assumed that the probing

16 tools were already well established, since they are found in many chimpanzee

17 populations, before the stout puncturing stick was invented. Another example from the

18 same site is the brush-tipped termite probe, where the regular termite probe (again

19 assumed to be the starting point, given its common presence in other populations)

20 undergoes an additional modification in which the tip is frayed, which makes it far more

21 effective in gathering termites (which bite into the probe, and latch on more easily if the

22 tip is frayed). This evidence from the wild is complemented by experimental work.

23 Recently, Lehner et al. (2011) coaxed captive orangutans into making ratcheted

24 innovations.

25 By these definitions, some chimpanzee technology in nature is cumulative,

26 although the majority is not, whereas captive orangutans can be coaxed into making it.

27 Thus, there is some overlap with the technology of the makers of the Oldowan (Table 1).

28 Nonetheless, whereas all orangutan and most tools in the wild are TL1 and some

29 chimpanzee tools are TL2 and perhaps even in one case TL3, regular Oldowan tools are

30 TL2, but Oldowan tools used to modify wooden tools are TL3. Acheulean tools, in

31 contrast, are TL3 or higher.

32 Given that great apes are now known to have sufficiently accurate powers of

33 observational learning to allow ratcheting and that they show some evidence of

34 cumulative technology in the wild or captivity, we need a new explanation of the major 
1 difference between humans and great apes in their technology. The goal of this paper is

2 therefore to identify the factors responsible for cumulative cultural evolution of

3 technology.

$4 \quad$ We begin by developing a model that correctly reproduces the known great ape

5 patterns. Modeling cumulative technology is made easier by the presence of

6 considerable variation among orangutans and chimpanzees, the two ape species

7 showing extensive tool use in the wild. Most orangutan populations fail to show any

8 systematic extractive tool use, but a few do and actually do so in multiple contexts (van

9 Schaik et al., 2003a), and even show some variation within populations, depending on

10 exposure to suitable role models (van Schaik et al., 2003b). All chimpanzee populations,

11 in contrast, show at least some tool use (Sanz and Morgan, 2007), and some, as noted

12 above, show evidence of ratcheting of technology. Having developed and tweaked the

13 model for great apes, we then examine the hominin case by changing the model's

14 parameter values in the direction of known or suspected changes during hominin

15 evolution.

\section{Methods}

18 In this paper we propose a novel simulation model to explain the process of 19 accumulation of technology. It is built using the same basic framework proposed by van 20 Schaik \& Pradhan (2003) to model tool use in great apes, which replicated geographic

21 variation in orangutan tool use, and found it to be a function of variation in opportunities

22 for social learning (see also Enquist et al., 2010). The current model simulates changes

23 in a population's level of technology over time, as a result of individual opportunities for

24 acquiring tool-use skills (either through invention or through social learning) at different

25 levels of accumulation (TLO through TL3). In the model, no skipping of technology levels

26 is allowed; thus, for instance, TL2 is a prerequisite for reaching TL3, both in the

27 innovation history and in ontogenetic acquisition. Even if this assumption does not

28 strictly hold during ontogeny and some skipping is allowed, it remains likely that learning

29 a skill is more difficult as TL increases.

30 We are interested in obtaining percentages of the total population engaged in

31 various technology levels at every time-step given suitable ecological conditions. The

32 model's parameters are listed and defined in Table 2, as are the best estimates of these

33 parameters for great apes (see below for justification of the actual values used). We

34 assume that every individual of the population has an intrinsic inventive ability $(\varepsilon)$. The 
1 parameter $\alpha$ refers to the probability that an individual learns the skill socially from any

2 of the nearest neighbors possessing higher TLs in a given year, subject to the constraint

3 that in a given year it can only move up one TL at best. Social learning can take place

4 because each individual in the population can be in tolerant proximity with some close

5 neighbors. In the model, each individual is represented as a node in a regular graph with

6 the degree $2 \kappa$ (Harary, 1969; $p$ 14), which is the number of close neighbors for each

7 individual. In such a social network, all the nodes are arranged on a circle. Individuals

8 can only learn from their nearest neighbors, i.e. the nodes to which they are directly

9 connected. The value of $\kappa$ is a function of the population's sociability, with high $\kappa$

10 indicating high social tolerance. Note that we do not consider the effect of the number of

11 demonstrators on skill acquisition through social learning. We chose this conservative

12 rule because it is not known how the presence of multiple demonstrators adds up, and in

13 the model most naïve individuals will often have only a single role model of a particular

14 TL anyway.

15 The total number of nodes gives the size of the population $(N)$. We could have

16 modeled the population as a two-tiered system, with subgroups that internally show tight

17 connections but are more loosely connected to other subgroups, but the effects would

18 not be systematically different, except in the time it takes to reach particular TL values

19 (see discussion).

20 For each combination of the parameters $\varepsilon, \alpha$, and $\kappa$, we calculated the

21 average fractions of the population that had attained the various technology levels after

22 a suitably large number of time steps. We think of each time step as representing one

23 year, which was also the time scale for the probabilities of innovation and acquisition

24 through social learning. Each individual starts life at technology level 0 (TLO). It can then

25 acquire higher technology levels through either personal innovation or social learning

26 until it dies (implemented as reverting back to level 0). In each time step, the annual

27 mortality rate $(\mu)$ sets the probability that the individual falls back to TLO and age 0 .

28 Each individual has a window of active social learning $(\lambda)$ that starts at birth and runs

29 until this value is reached, representing the age at which it stops to learn socially, but

30 after which it can still serve as role model. We also inserted a maximum age $(M)$,

31 although virtually no individual ever reached this. This structure produced a constant

32 population that is roughly age-structured, but without having to introduce actual ages or

33 other unrealistic simplifications. 
As illustrated in the flow chart (Figure 1), the simulation goes through the

2 following steps. We generate the social graph with degree $2 \kappa$. We then initialize the

3 model at technology level 0 (TL0) for all individuals, who are initially all at age 0 . In each

4 time step (year), we sequentially check all nodes, and for each node, we check their

5 survival. If the node survives and is within the age range in which it is capable of social

6 learning, we check whether the node reaches the next TL through invention, and if not,

7 whether it reaches the next TL through social learning. When all the nodes are

8 traversed, we calculate the proportion of the population with TLO through TL3 (or even

9 higher TLs, see below). This procedure is then repeated for 10,000 steps, although

10 equilibrium is almost always reached well before this time. To account for statistical

11 variation in individual outcomes, the results reported in the following graphs are

12 averages of 200 realizations of this procedure.

14 Parameter estimates for great apes

15 Because the model contains many parameters, high TL can be reached through

16 many combinations. Thus, in order to increase the explanatory power of the simulations

17 for hominins, we first determined the most realistic ranges for each parameter among

18 great apes. We base these on the well-studied orangutan case of Neesia tool use (van

19 Schaik and Knott, 2001; van Schaik, 2009), although we also examined the effect of

20 each parameter over the whole range of values.

21 Starting with $N$, and given that orangutan populations do not consist of loosely

22 connected subgroups, $N$ is realistically in the range of hundreds or more, since most

23 natural populations are in the range of up to a few thousand (Singleton et al., 2004). We

24 kept values of $\alpha$ rather low (around 0.2). Great apes may be able to copy, but they are

25 not good copiers (see above) and many activities are not performed long enough for

26 much observational learning to occur, explaining why some profitable innovations

27 originate but do not seem to spread (e.g. Yamamoto et al., 2008). In addition, many

28 aspects of the techniques cannot be seen and must be acquired by individual practice,

29 which is bound to take time (see also Stout, 2011 for humans). Finally, immatures take

30 several years of observation alternated with practice to acquire tool-use techniques

31 (Matsuzawa et al., 2001; Lonsdorf et al., 2004; van Schaik, 2004; E. Meulman, in prep.).

32 Therefore, it is unlikely that $\alpha$ is much higher than 0.2 in ape populations for the more

33 rarely invented skills whose origin and spread is modeled here. Obviously, for simpler

34 skills, $\alpha$ levels could well be far higher, but here we are modeling the most complex 
1 technology shown by these animals in the wild. In a more conservative version of the

2 model, one could reduce $\alpha$ levels, as TL increases, but this would not qualitatively

3 change the outcome of the simulations (results not presented).

$4 \quad$ We put $\kappa$ at low levels, since even in the most sociable orangutan populations,

5 which have Neesia tool use, immatures do not associate with others than the mother

6 more than about $60 \%$ of time, and although only weaned individuals are counted as

7 associates, not all of these are fully adult (van Noordwijk et al., 2009). Since many

8 associations are with a single independent individual only, there are about 2 nearest

9 neighbors on average in these populations, giving $\kappa=1$.

10 Given that we tried to replicate the distribution of Neesia tool use first, we

11 decided that the innovation rate, $\varepsilon$, should be low, reflecting the absence of tool use in

12 many populations inhabiting primary forests with Neesia trees, where it could potentially

13 be seen (van Schaik, 2009). Since both seed extraction and nut cracking provide more

14 calories than any other activity in these great ape populations (van Schaik and Knott,

15 2001), we must assume that individuals that invented this skill would keep on using it. By

16 putting $\varepsilon=0.0001$, we in effect assume that one in 500 individuals (given a mean annual

17 mortality rate of 0.05 , and thus an average lifespan of 20 years) will independently invent

18 the technique, suggesting that in a large population of 500 , one individual on average

19 has come up with it. At $\varepsilon=0.001$, this probability is still one in 50 , so high that tool use

20 should be seen in most orangutan populations with Neesia fruits. Since it is not, $\varepsilon$

21 should realistically be in the order of 0.0001 or less.

22 Sumatran orangutans can live up to at least 50 years in the wild (Wich et al.

23 2004), so this was the maximum age $(M)$ in the simulation. Annual mortality rates $(\mu)$

24 were set at 0.05 , which may be somewhat high for orangutans (Wich et al., 2004), but is

25 certainly at the low end for chimpanzees (Hill et al., 2001).

26 Orangutans do not effectively use tools until weaning age (roughly 7 years; van

27 Schaik, 2004), and once adult may, like chimpanzees, gradually lose interest in learning

28 in general, and social learning in particular (e.g. Tomasello et al., 1987; Matsuzawa et al.

29 2001; Tennie et al., 2010; Hobaiter and Byrne, 2010). Given that they may not be

30 effective at learning complex techniques for the first year or two, this suggests that the

31 social-learning window $(\lambda)$ is in the order of 15 years or somewhat more. This is a rough

32 estimate, because we do not have good estimates of how age affects social learning

33 ability or interest. 


\section{Results}

2 General model results

3 The model's output is the percentage of the population that has reached technology

4 levels TL0, 1, 2, or 3, as a function of time (in years). In Figure 2.a, we plot this for a

5 hypothetical great-ape population with moderate sociability ( $N=501, \alpha=0.2, \kappa=1$,

$6 \varepsilon=0.0001, \mu=0.05, \lambda=15)$. At this level of sociability, TL1 can establish itself. In

7 Figure 2.b, we have increased sociability to $\kappa=2$. Now, TL1 establishes itself first,

8 peaks, and then gives way to TL2, which in turn gives way to TL3. However, both TL1

9 and TL2 also stay around at low levels, reflecting the presence of individuals that have

10 not yet reached the higher TL. This is therefore an example of a population in which

11 ratcheted technology did evolve. We also examine the case where sociability is

12 moderate $(\kappa=1)$, but innovation rate is two orders of magnitude higher than what we

13 think is the case for the normal great ape situation $(\varepsilon=0.01)$. Figure 2.c shows that only

14 a few members of the population reach TL3. Thus, the rate of innovation itself has far

15 less influence on the TL reached by the average population member than the nature of

16 the transmission conditions.

18 Great apes and cumulative technology

19 Orangutans and chimpanzees have similar cognitive abilities (Deaner et al.,

20 2006; Herrmann et al., 2007; Tennie et al., 2010), and hence similar $\varepsilon$ and $\alpha$. They also

21 have comparable life histories (Hill et al., 2001; Wich et al., 2004), and hence similar

22 mortality rates $(\mu)$ and windows for social learning $(\lambda)$, leaving only sociability $(\kappa)$ and

23 population size $(N)$ to show enough geographic variation to affect variation in

24 technology.

25 Figure 3 shows the results when the parameters were held constant at the values

26 selected above (Fig. 2), but sociability, $\kappa$, corresponding to the number of possible

27 tolerant experts in social learning, was varied. As expected, there is a strong effect of $\kappa$.

28 The range from 0.5 (solitary, with mother as sole companion) to 2 (on average

29 surrounded by 4 knowledgeable models, one of whom is the mother) correctly

30 reconstructs the observed patterns among great apes (van Schaik et al., 2003a). In the

31 most solitary situation ( $\kappa=0.5$ ), corresponding to the orangutans in most of Borneo, we

32 see virtually no TL1, and nothing higher, even after many years (here, 10,000). The

33 situation with $\kappa=1$, corresponding to the social situation in orangutans inhabiting Suaq 
1 and other swamps on Sumatra's west coast but also many chimpanzee populations,

2 leads to customary TL1, with the odd individual reaching TL2. Once $\kappa$ reaches 2, we

3 see that TL2 or even TL3 become customary, but it is not clear whether any known

4 chimpanzee populations reach this level of sociability.

$5 \quad$ The degree of ratcheting that can be achieved by great apes is probably limited

6 by the "affordance forcing" effect of the raw materials (see Table 1). This effect is

7 expressed in the limited variability in great ape tools and the strict form-function

8 correlation among these tools. These features reflect the biases in cognitive capacities

9 of the species in question (the "latent solutions"), which evolved to detect and deal with

10 the affordances of objects in the presence of suitable problems (Tennie et al. 2009).

11 Affordance forcing limits the amount of accumulation that can be achieved with the same

12 raw materials, justifying stopping the simulations at a moderate level, e.g. TL3; it is also

13 the reason why in our model we do not consider distortions in techniques, which would

14 have arisen due to error-prone copying. However, if we allow TL to increase, at $\kappa=2$, in

15 equilibrium a considerable proportion of the population had reached $T L 4$, underlining the

16 very strong effect of sociability on ratcheting. Thus, the main finding is that increased

17 sociability will lead to ratcheting, and thus higher TL, among great ape populations.

18 The effect of $N$, in contrast, is entirely one of timing (Figure 4). At higher $N$ the

19 various TL levels are reached after a shorter number of generations, but the equilibrium

20 levels are identical. Thus, in this model, $N$ has no effect on the presence of the degree of

21 accumulation of a particular technique, except in unusually small and isolated

22 populations. This implies that a major demographic effect is expected only if populations

23 frequently go extinct and areas are re-colonized by culturally naïve individuals, initially in

24 small populations. Both the presence for over 4,000 years of nut cracking based on

25 stone tools in chimpanzees (Mercader et al., 2002) and the unusually long temporal

26 stability of orangutan populations in northern Sumatra (Nater et al., 2011) suggest that

27 such demographic constraints were not ubiquitous. Moreover, known major habitat

28 changes, such as recovery from the last glacial period, happened thousands, not

29 hundreds, of years ago, long enough to produce equilibrium levels of cumulative

30 technology. However, frequent local extinctions may have been an important factor in

31 isolated populations or regions at the edge of the geographic distribution.

32

33 Hominin evolution until early Homo 
1 Having successfully reconstructed the pattern of accumulation observed among great

2 apes, we now examine whether the cultural changes in hominins with lithic technology

3 could be a reflection of simple, immediate changes in socioecology. We do this by

4 extending the standard great ape model as used above into parameter values known to

5 characterize hominins. Morphological and archeological features suggest changes in

6 sociability, social learning through teaching, and terrestriality.

$7 \quad$ The first evidence of routine butchering of large carcasses was around by $2.5 \mathrm{Ma}$

8 (Semaw et al., 1997; de Heinzelin et al., 1999), in a way that suggests the presence of

9 societies with unusually high sociability, needed to either acquire the prey through

10 hunting or defend the carcasses against large carnivores (confrontational scavenging).

11 This suggests a clear increase in $\kappa$, which is a reliable way to get a hominin population

12 to reach higher TL levels (Figure 3). At $\kappa=3$, we see that all adults reach TL3, well

13 beyond what extant great apes can achieve. Allowing TL to move up freely yields a

14 maximum at TL6, provided the affordances of the raw materials allow this.

15 There are many indications that extensive allomaternal care in the form of

16 systematic food sharing or even provisioning, similar to what is seen among

17 cooperatively breeding animals, began after the routine deployment of cooperative

18 hunting subsequent to $2.5 \mathrm{Ma}$, and was firmly established by the appearance of Homo

19 erectus around 1.7 Ma (Hrdy, 2009; van Schaik \& Burkart, 2010; K. Isler and C. van

20 Schaik, in review). Therefore, the demonstrators (already more numerous than earlier)

21 probably also gradually began to engage in teaching, since teaching is common among

22 cooperatively breeding animals with complex foraging techniques (Hoppitt et al., 2008;

23 Rapaport and Brown, 2008; Burkart and van Schaik, 2010). Teaching, by definition,

24 raises the social-learning ability, $\alpha$. Figure 5 shows the effect of raising $\alpha$, at two levels

25 of sociability, $\kappa$. An increase of $\alpha$ beyond the level seen in great apes (for instance as a

26 result of opportunitiy teaching, largely amounting to providing opportunities for

27 appropriate practice: Caro and Hauser, 1992) provides a strong boost to technological

28 accumulation. At higher levels of $\kappa$, this effect reaches a ceiling, when we keep TL fixed

29 at 3 . Nonetheless, in less than 1,000 years, such a population, despite having great-ape

30 level cognitive abilities, will reach the maximum TL3 level. If we allow TL to evolve freely,

31 a population with $\kappa=3$ and $\alpha=0.4$ will reach a maximum TL10, showing considerable

32 ratcheting potential, probably well beyond what actually was possible with Oldowan

33 techniques (see Table 1). 
The processing of large animal carcasses necessarily took place on the ground.

2 Among primates, systematic terrestriality can be shown to affect technological evolution

3 because it leads to closer proximity (and thus higher $\kappa$ ) and systematic opportunities for

4 affordance learning of the technology (and thus higher) (Meulman et al., in press). By the

5 time Homo erectus appeared, these hominins had become systematically terrestrial

6 (Bramble and Lieberman, 2004) and probably performed all skilled activities on the 7 ground.

\section{Discussion}

10 Implications of the model

11 By ca $2.5 \mathrm{Ma}$, hominins already had reached lithic technology levels exceeding

12 that of most chimpanzees (see Table 1), showing a definite advance toward cumulative

13 technology. The simulations presented here suggest that this development in hominins

14 was induced by changes in social organization that led to higher sociability, brought

15 about by cooperative hunting or scavenging, followed by the adoption of full terrestriality

16 and teaching elicited by systematic food sharing and provisioning, which further

17 improved social transmission of skills. By the time Homo erectus appeared, some time

18 before $1.7 \mathrm{Ma}$, the relevant social parameters (sociability $\kappa$, social learning ability $\alpha$ )

19 had reached values that in the model guarantee stable cumulative technology.

20 The simulations imply that considerable technological accumulation can be

21 achieved without any increase in innovation rate, population size, or development time

22 (slower-paced life history). The fossil and archeological records also support this

23 conclusion. First, the strong correlations between brain size and innovation ability in both

24 birds and mammals (Lefebvre et al., 2004; Reader and Laland, 2002; Deaner et al.,

25 2007) suggest that brain size can be taken as predictive of innovation level, $\varepsilon$. The

26 Oldowan (Semaw et al., 1997) appeared well before major increases in brain size

27 (Schoenemann, 2006). Indeed, our simulations show no effect of increased $\varepsilon$ on the

28 degree of accumulation of a particular technique (Figure 6, in which we assume $\alpha=0.4$

29 and $\kappa=2$ ), except that it speeds up the time at which maximum TL is reached (similar to 30 the effect of $N$ ). Second, these hunting or scavenging hominins occupied higher levels in

31 the trophic pyramid, and their population sizes are therefore likely to have been smaller

32 than those of extant great apes. However, although higher population size or

33 connectivity would have helped, they were not required: In smaller populations the same

34 development (ratcheting of technology) would have reached the same equilibrium level, 
1 but would merely have taken longer. The time scale of changes observed in the

2 archeological record indicates a very slow pace of change (Klein, 2009). Finally, the

3 scant data on the life history of hominins predating Homo erectus, as deduced from

4 tooth development (G. Schwartz, in prep.), indicate faster development than among

5 extant great apes, and hence higher, rather than lower, mortality.

6 The model therefore strongly suggests that the first stages of cumulative

7 technology required no increases in cognitive abilities (abilities to innovate or learn

8 socially), because populations could achieve higher ratcheting levels so long as the

9 essential innovations arose with some non-zero probability and were passed on with

10 some non-zero probability. Obviously, these cultural changes were themselves likely to

11 have served as the impetus for the subsequent evolution of greater cognitive abilities,

12 especially via improved social-learning abilities, which secondarily improved innovative

13 capacity as well (Wyles et a., 1983; van Schaik and Pradhan, 2003; Whiten and van

14 Schaik, 2007; van Schaik and Burkart, 2011).

15 These results may appear to contradict the results of theoretical work on recent

16 cultural accumulation in human evolution (Culotta, 2010), which stress the role of

17 population expansion (Powell et al., 2009) and cognitive changes leading to increased

18 innovation (Coolidge and Wynn, 2009). However, the pace of cultural change in (what is

19 now visible in) the Oldowan and Acheulean was so slow that population size, which

20 affects the time it takes for specific innovations to become established, hardly mattered.

21 Moreover, increased innovation rate is both a direct and indirect outcome of

22 accumulation itself. Thus, in the Middle and Upper Paleolithic, other processes may

23 have determined cultural evolution.

24 The model's results also would suggest that considerable ratcheting of

25 technology should have been possible, well beyond the Oldowan technology seen in the

26 record for well over a million years, and should also still encompass the early Acheulean

27 (cf. Table 1). This discrepancy may be more apparent than real because of the

28 limitations of the archeological record, which does not retain any use of plant-based

29 tools, alone or in combination with stone tools. Indeed, the greatest complexity would be

30 reached for tool sets, functional sequences of (usually fairly simple) tools, as seen in the

31 chimpanzees, but they likewise cannot be recognized in the archeological record.

32 However, if the discrepancy is real, this implies that our model cannot be applied to later

33 stages of hominin evolution. The most likely reason for this is that neither great apes nor

34 early hominins could reach higher TL than the moderate levels (TL3 or TL4) examined in 
1 this model, due to limits on the amount of ratcheting imposed by the nature of the raw

2 materials in combination with the cognitive biases (see above), or the increased

3 cognitive difficulty of producing further modifications due to constraints on working

4 memory.

\section{Model assumptions}

7 The model results obviously depend on our operationalization of ratcheting. First, it 8 assumes, with Davidson and McGrew (2005) and Wynn et al. (2011), that the cognitive

9 challenges posed by stick tools and stone tools both depend on the technology level

10 (TL) and thus the depth of the planning hierarchy, despite clear differences in the kinds 11 of actions performed on sticks and stones. Thus, a clear prediction is that the same 12 population of a given species should reach similar TL in different kinds of technology.

Table 1 might seem to indicate that this assumption is wrong, as chimpanzees,

14 the only great ape species to use any stone tools in the wild, do not reach higher TL in

15 such tools, in stark contrast to stick tools, which have ratcheted up to TL3 in the wild.

16 However, the discrepancy may only be apparent. Wild chimpanzees are doubly

17 disadvantaged when it comes to ratcheting stone tools. Chimpanzees lack the need for

18 artificial sharp edges (their teeth are long, strong and sharp enough for the problems

19 they face, as pointed out by Davidson and McGrew (2005) as well as Toth and Schick

20 (2009)), and even if they would discover the need for sharp tools, their environment

21 largely lacks the suitable stone resources that could be knapped (Carvalho et al., 2008).

22 It is therefore important to explore the capacity of captive apes. As it happened,

23 beside a pioneer study on one orangutan (Wright 1972) all work on great ape flaking

24 focused on bonobos, not chimpanzees. Kanzi, the main tested bonobo developed,

25 perfected, and later preferred, to throw the stones given to him against each other in

26 order to produce blades. He developed this preference over the course of only 120

27 hours of experience (Schick et al. 1999). Importantly, Kanzi invented the throwing

28 technique without having seen it modeled (thus, this is clearly a behavior within the

29 bonobo ZLS) - and which is a behavior that remains to be analyzed in complexity and

30 compared to two-handed flint knapping, in the way proposed by Bril et al. (2012).

31 Certainly, the products thus produced were not fully comparable to Oldowan artifacts.

32 Bril et al. take this to mean that Oldowan flint knapping included some more complex

33 behaviors than we can find in modern great apes, but did not consider the possibility that

34 Kanzi failed in this respect perhaps because he was biomechanically restricted (Toth 
1 and Schick, 2009) in a way that chimpanzees might not be. Moreover, a female bonobo

2 called Panbanisha was observed "making stone tools, and she appeared to calculate

3 angles before hitting the core" (Davidson \& McGrew, 2005). It was also not the case that

4 Kanzi could not flake in a hand-held, bimanual way. Kanzi may have simply realized that

5 - for him - to flint knap in a two-handed way was less efficient than the throwing

6 technique; perhaps not so much for cognitive, but perhaps merely for anatomical 7 reasons.

8 Given the higher proficiency of tool use of chimpanzees in the wild in contrast to

9 bonobos (e.g. Whiten et al., 1999), the best way to definitively test Bril et al.'s (2012)

10 hypothesis is to test chimpanzees. At present, all we have is an unpublished study by

11 Sarah Boysen and her team, who for the first time have provided chimpanzees with the

12 need (a reward box with a rope-lock) as well as the necessary raw material to flint knap

13 (a granite hammer stone and a raw flint rock). After two short demonstrations, one of the

14 two tested chimpanzees flint knapped with high proficiency - at a level that, at

15 preliminary analysis, clearly outperformed previously tested bonobos (S. Boysen, pers.

16 communication, 12th July 2011). The resulting sharp edge was immediately used to cut

17 through the rope that hindered the chimpanzee to get the reward. This to us shows the

18 higher potential for flint knapping in chimpanzees in contrast to bonobos. Thus, the

19 chimpanzee may not only have the cognitive capacity for Oldowan-like tools, it may also

20 have the motor control to do so (somewhat in contrast, perhaps, to the case of

21 bonobos), consistent with the critical assumption of our modeling study (cf. Wynn et al.,

22 2011).

23 A second testable prediction of this operationalization of ratcheting is that

24 developing individuals should make simpler versions of tools before they make the more

25 ratcheted ones. This would seem to hold true of currently living great apes and extinct

26 hominins. Thus, we predict that immature chimpanzees should learn to make termite

27 probes first, and then later on learn how to fray the sticks' ends to make them brushy,

28 rather than to learn all of this at once (or if they do learn it all at once, they must learn it

29 after longer practice, so probably at a later age than those in other populations that use

30 simple probes). A third testable prediction is that ratcheted tools should have a more

31 limited geographic distribution, and should often be nested inside the region of

32 'ancestral' simple forms from which they derive. Finally, it is to be hoped that

33 neurobiological correlates of ratcheting can be revealed, which allow comparisons

34 across species and tasks. 
Future work should therefore test the model's assumptions. This may lead to

2 modified conclusions. However, Sanz \& Morgan's (2010) recent attempt to apply

3 different systems of accounting for complexity to tools used by Goualougo chimpanzees

4 revealed only modest discrepancies between them. Future work should thus also show

5 to what extent classifications of complexity must also incorporate the choice of raw

6 materials and the complexity of actions (Haidle, 2010; Stout, 2011).

7 Apart from the measurement of ratcheting, the model also assumed a role for

8 sociability, the number of available role models. As an earlier model (van Schaik and

9 Pradhan, 2003) predicted, evidence can indeed be found for enhanced culture in groups

10 of great apes that are more sociable (Whiten and van Schaik, 2007). However, to the

11 best of our knowledge the parameters identified here as the major ones for the

12 accumulation of culture, have not yet been measured systematically in wild great ape

13 populations and subsequently been correlated with the amount of ratcheting observed

14 (let alone manipulated experimentally). This may also be due to the fact that

15 accumulation is rare in great apes, and that thus any such correlation is hard to

16 establish. We hope that future work in both field and captivity will test these various

17 predictions.

Technological Evolution

The results of this study suggest a new perspective on the nature and timing of

21 the major transitions in the cultural evolution of technology in primates and humans

22 (Figure 7). The first phase began with the origin of simple culturally based technologies.

23 It required the presence of extractive foraging and some cognitive abilities, enabling

24 innovation and social learning (van Schaik et al., 1999). This level is reached by many

25 great ape and some monkey (Ottoni and Izar, 2008; Gumert et al., 2009) populations, as

26 well several non-primate lineages of mammals and birds (Whiten and van Schaik, 2007).

27 The second phase began with the origin of ratcheted technology. This can be

28 elicited in captive apes and is found among some chimpanzee populations (and by

29 inference early hominins), and is routinely present in late Pliocene and early Pleistocene

30 hominins. We suggested here that this transition was made possible by increased

31 sociability and terrestriality, and subsequently teaching (see also Tomasello 2009).

32 However, this process probably also reaches some ceiling, set by material, cognitive

33 (especially size of working memory), demographic and life-history limitations, reached by

34 early Homo. The complexity of each particular technique may also reach a ceiling 
1 because an individual may have to learn various different techniques simultaneously,

2 and thus cannot fully concentrate on any single technique. It may be difficult to break

3 through this ceiling because each individual must learn to produce all the technology

4 used in its society and is therefore constrained by learning time (although this gets

5 longer as life-history pace slows down, and especially as the learning window expands).

6 It is still possible, of course, that adaptations could have enabled hominins to move

7 beyond this ceiling. For instance, lengthening the duration of the learning period $(\lambda)$ will

8 help. Nonetheless, some limitation should emerge, and our model strongly suggests that

9 the prime mover was not cumulative culture per se.

10 Because of the constraints on learning time, we propose a third transition in

11 cultural evolution to explain levels of accumulation beyond the ones we have modeled

12 here. Moving beyond the results of our models, we speculate that it was caused by the

13 appearance of donated technology, which relied on two major components: technology

14 transfer and specialization (Figure 7). In technology transfer, naïve individuals would

15 receive implements or concepts that they did not invent and could not design and

16 produce themselves, allowing them to skip many steps in the ratcheting process and use

17 these technologies to invent further ratcheted technologies. Thus, here we can begin to

18 encounter cumulative culture in the sense of the appearance and subsequent routine

19 use of innovations well beyond the innovative reach of individuals (cf. Galef, 1992;

20 Tomasello, et al., 1993; Boyd and Richerson, 1996). Specialization, or advanced division

21 of labor, means that individuals no longer need to learn all the technology used in a

22 particular society, but can instead focus on acquiring a particular subset of skills. This

23 almost inevitably produces higher technology levels in the population as well.

24 Specialization can be recognized ethnographically by examining the TL level at which

25 requests for help with the repair of tools and implements are made. One can also

26 recognize it in the archeological record with the onset of long-distance trade (which

27 presupposes specialization, unless the traded items were naturally occurring resources

28 that were traded prior to processing them).

29 Both these novel features imply larger societies with a high level of cooperation

30 and enough of a food surplus to support specialists that are not full-time food producers.

31 Moreover, the high technology implies at least part-time sedentism and presumably

32 trading of specialist products.

33 Because individuals can skip technology levels by using implements produced by

34 other experts, and because different specialists acquire different skills, this third phase 
1 has a far greater potential to produce run-away cumulative technology. Hence, it is in

2 this phase that demographic limitations (and therefore also time) become important, as

3 stressed by recent archeological models that focus on relatively recent changes in the

4 technology of Homo sapiens (Shennan, 2001; Henrich, 2004; Powell et al., 2009).

\section{Conclusions}

$7 \quad$ This modeling study showed that we could explain variation among orangutans

8 and chimpanzees in the presence and degree of accumulation of their (mainly wood-

9 based) technology with reference to varying sociability, which affects the opportunities

10 for social learning. The degree of accumulation of technology well into levels shown by

11 the most complex Oldowan tools can plausibly be attributed to further increases in

12 sociability, and the introduction of teaching, which increases the probability of acquiring

13 a skill through social learning. Thus, according to the model, no major cognitive

14 changes, relative to extant great apes, were needed to explain the origin and initial

15 elaboration of lithic technology in the hominin lineage, consistent with the observation

16 that the two taxa had similar brain sizes. Our model also indicates that population

17 (network) size is less important than previously thought - though it can be still important

18 in timing, especially with regard to the rate of environmental change (d'Errico and

19 Stringer, 2011). However, once populations consistently began to have highly ratcheted

20 technology, selection may have begun to favor enhanced cognitive abilities, allowing

21 faster developmental acquisition and (as a byproduct) the innovation of more complex

22 techniques. Indeed, by the time the Acheulean appeared, hominin brain sizes exceeded

23 those of extant great apes. This argument suggests that the seemingly autocatalytic

24 increase in brain size during the early evolution of Homo was driven by technological

25 evolution rather than by other factors such as social complexity per se.

26 Because considerable cumulative cultural evolution is possible with great-ape-

27 sized brains, as implied by results form the wild and experiments in captivity, the

28 ratcheted technology of hominins should no longer be considered qualitatively unique,

29 although they subsequently pushed it to much higher levels than the extant great apes.

30 We speculate that a truly qualitatively change in technological evolution came much

31 later, in the form of donated technology, when individuals could use the products of

32 others' efforts as their starting point, allowing them to skip many steps in the learning

33 process, and individuals could also specialize in acquiring particular subsets of the skills

34 present in the population as a whole. 


\section{Acknowledgments}

3 We thank Sagar Pandit for helpful discussions. We also thank the anonymous reviewers

4 for their suggestions and questions. Supported by Swiss National Fund (grant no.

5 31003A-111915), the A.H. Schultz Foundation, and the Max Planck Society. 


\section{REFERENCES}

2 Biro, D., Inoue-Nakamura, N., Tonooka, R., Yamakoshi, G., Sousa, C., Matsuzawa, T., 2003. Cultural innovation and transmission of tool use in wild chimpanzees: evidence from field experiments. Anim. Cogn. 6, 213-223.

Boesch, C., Marchesi, P., Marchesi, N., Fruth, B., Joulian, F., 1994. Is nut cracking in wild chimpanzees a cultural-behavior? J. Hum. Evol. 26, 325:338.

Boesch, C., 2003. Is culture a golden barrier between humans and chimpanzee? Evol. Anthropol. 12, 82-91.

Boesch, C., Head, J., Robbins, M.M., 2009. Complex tool sets for honey extraction among chimpanzees in Loango National Park, Gabon. J. Hum. Evol. 56, 560-568.

Boyd, R., Richerson, P.J., 1996. Why culture is common, but cultural evolution is rare. Proc. Brit. Acad. 88, 77-93.

Bramble, D.M., Lieberman, D.E., 2004. Endurance running and the evolution of Homo. Nature 432, 345-352.

Bril, B., Smaers, J., Steele, J., Rein, R., Nonaka, T., Dietrich, G., et al. 2012. Functional mastery of percussive technology in nut-cracking and stone-flaking actions: experimental comparison and implications for the evolution of the human brain. Philosophical Transactions of the Royal Society B:

20 Burkart, J.M., van Schaik, C.P., 2010. Cognitive consequences of cooperative breeding in primates. Anim. Cogn. 13, 1-19.

\section{Byrne, R.W., 1995. The Thinking Ape: Evolutionary Origins of Intelligence. Oxford} University Press, Oxford.

Byrne, R.W., Tanner, J.E., 2006. Gestural imitation by a gorilla: Evidence and nature of the capacity. Intern. J. Psych. Psychol. Therapy 6, 215-231.

Caro, T.M, Hauser, M.D., 1992. Is there teaching in nonhuman animals? Q. Rev. Biol.

28 Carvalho, S., Cunha, E., Sousa, C., Matsuzawa, T., 2008. Chaines operatoires and resource-exploitation strategies in chimpanzee (Pan troglodytes) nut cracking. J. Hum. Evol. 55, 148-163.

Claidière, N., Sperber, D., 2010. Imitation explains the propagation, not the stability of animal culture. Proc. Roy. Soc., B. 277, 651-659.

Coolidge, F.L., Wynn, T. 2009. The Rise of Homo sapiens: The Evolution of Modern Thinking. Wiley-Blackwell, Oxford. 
1 Culotta, E., 2010. Did modern humans get smart or just get together? Science, 328, 164164.

3 d'Errico F., Stringer C. B., 2011. Evolution, revolution or saltation scenario for the emergence of modern cultures? Phil. Trans. Roy. Soc. B. Biological Sciences, 366, 1060-1069.

Davidson, I., McGrew, W. C., 2005. Stone tools and the uniqueness of human culture. Journal Royal Anthropological Institute 11:793-817.

8 Deaner, R.O., van Schaik, C.P., Johnson, V., 2006. Do some taxa have better domaingeneral cognition than others? A meta-analysis of nonhuman primate studies.

Deaner, R.O., Isler, K., Burkart, J.M., van Schaik, C.P., 2007. Overall brain size, and not encephalization quotient, best predicts cognitive ability across non-human primates. Brain Behav. Evol. 70, 115-124.

de Heinzelin, J., Clark, J. D., White, T., Hart, W., Renne, P., WoldeGabriel, G., Beyene, Y., Vrba, E., 1999. Environment and behavior of 2.5-Million-year-old Bouri hominids. Science 284, 625-629.

Dindo, M., Stoinski, T., Whiten, A., 2011. Observational learning in orangutan cultural transmission chains. Biol. Lett. 7, 181-183.

Dominguez-Rodrigo, M., Serrallonga, J., Juan-Tresserras, J., Alcala, L. \& Luque, L. 2001. Woodworking activities by early humans: a plant residue analysis on Acheulian stone tools from Peninj (Tanzania). J. Hum. Evol. 40, 289-299.

Enquist, M., Strimling, P., Eriksson, K., Laland, K.N., Sjostrand, J., 2010. One cultural parent makes no culture. Anim. Behav. 79, 1353-1362.

Galef, B.G.-Jr., 1992. The question of animal culture. Hum. Nature 3, 157-178.

Gruber, T., Muller, M.N., Strimling, P., Wrangham, R., Zuberbuhler, K., 2009. Wild chimpanzees rely on cultural knowledge to solve an experimental honey acquisition task. Curr. Biol. 19, 1806-1810.

Gumert, M. D., Kluck, M., Malaivijitnond, S., 2009. The physical characteristics and usage patterns of stone axe and pounding hammers used by long-tailed macaques in the Andaman Sea region of Thailand. Am. J. Primatol. 71, 594-608.

Haidle, M.N., 2010. Working-memory capacity and the evolution of modern cognitive potential: implications from animal and early human tool use. Curr. Anthro. 51, S149-S166.

Harary, F., 1969. Graph Theory. Reading, MA: Addison-Wesley Publishing Company. 
Henrich, J., 2004. Demography and cultural evolution: how adaptive cultural processes can produce maladaptive losses - The Tasmanian case. Am. Antiq. 69, 197-214.

Herrmann, E., Call, J., Hernandez-Lloreda, M.V., Hare, B., Tomasello, M., 2007. Humans have evolved specialized skills of social cognition: The Cultural Intelligence Hypothesis. Science 317, 1360-1366.

Hill, K., Boesch, C., Goodall, J., Pusey, A., Williams, J., Wrangham, R., 2001. Mortality rates among wild chimpanzees. J. Hum. Evol. 40, 437-450.

Hill, K., 2009. Animal "Culture". In: Laland, K.N., Galef, B.G. (Eds) The Question of Animal Culture, Harvard University Press, Cambridge, MA, pp. 269-287.

Hobaiter, C., Byrne, R.W., 2010. Able-bodied wild chimpanzees imitate a motor procedure used by a disabled individual to overcome handicap. PLoS One 5 , e11959.

Hoppitt, W.J.E., Brown, G.R., Kendal, R., Rendell, L., Thornton, A., Webster, M.M., Laland, K.N., 2008. Lessons from animal teaching. Trends Ecol. Evol. 23, 486-493. Hrdy, S., 2009. Mothers and others: The evolutionary origins of mutual understanding. Harvard University Press, Cambridge, MA.

Jaeggi, A., Dunkel, L., van Noordwijk, M.A., Wich, S.A., Sura, A.A.L., van Schaik, C.P., 2010. Social learning of diet and foraging skills by wild immature Bornean orangutans: implications for culture. Am. J. Primatol. 72, 62-71.

Klein, R., 2009. The Human Career: Human, Biological and Cultural Origins, Third edn. Chicago University Press, Chicago.

Krützen, M., Willems, E.P., van Schaik, C.P., 2011. Culture and geographic variation in orangutan behavior. Current Biology 21:1808-1812.

Lefebvre, L., Reader, S.M., Sol, D., 2004. Brains, innovations and evolution in birds and primates. Brain Behav. Evol. 63, 233-246.

Lehner, S.R., Burkart, J.M., van Schaik, C.P. (2011) Can captive orangutans (Pongo pygmaeus abelii) be coaxed into cumulative build-up of techniques? J. Comp. Psych. Advance online publication

Lonsdorf, E.V., Eberly, L.E., Pusey, A.E., 2004. Sex differences in learning in chimpanzees. Nature 428, 715-716.

Matsuzawa, T., 1996. Chimpanzee intelligence in nature and in captivity: isomorphism of symbol use and tool use. In: C. MW, Marchant LF, Nishida T (Eds) Great ape

34 Matsuzawa, S., Biro, D., Humle, T., Inoue-Nakamura, N., Tonooka, R., Yamakoshi, G., 
2001. Emergence of culture in wild chimpanzees: Education by masterapprenticeship. In: Matsuzawa, S. (Ed) Primate Origins of Human Cognition and Behavior. Springer, Tokyo, pp. 557-574.

Meulman, E.J.M., Sanz, C.M., Visalberghi, E., van Schaik, C.P., (in press) The role of terrestriality in promoting primate technology. Evol. Anthropol.

Mercader, J., Panger, M., Boesch, C., 2002. Excavation of a chimpanzee stone tool size in the African rainforest. Science 296, 1452-1455.

8 Nater A., Nietlisbach, P., Arora, N., van Schaik, C. P., van Noordwijk, M. A., Willems, E. Perwitasari-Farajallah, D., Pamungkas J., Krutzen M., 2011. Sex biased dispersal and volcanic activities shaped phylogeographic patterns of extant orangutans (genus: Pongo). Mol. Ecol. Evol. 28, 2275-2288.

Nonaka T., Bril B., Rein R., 2010. How do stone knappers predict and control the outcome of flaking? Implications for understanding early stone tool technology. J. Hum. Evol. 59, 155-167.

Ottoni, E., Izar, P., 2008. Watching the best nutcrackers: what capuchin monkeys (Cebus apella) know about others' tool-using skills. Evol. Anthro. 17, 171-178.

Oswalt, W.H., 1976. An Anthropological Analysis of Food-Getting Teechnology. John Wiley, New York.

Powell, A., Shennan, S., Thomas, M.G., 2009. Late Pleistocene demography and the appearance of modern human behavior. Science 324, 1298-1301.

Price, E. E., Lambeth, S. P., Schapiro, S. J., Whiten, A., 2009. A potent effect of observational learning on chimpanzee tool construction. Proc. Roy. Soc., B. 276, 3377-3383.

Rapaport, L.G., Brown, R.B. 2008, Social influences on foraging behavior in young nonhuman primates: Learning what, where, and how to eat. Evol. Anthro. 17, 189201.

Reader, S.M., Laland, K.N., 2002. Social intelligence, innovation and enhanced brain size in primates. Proc. Natl. Acad. Sci., USA 99, 4436-4441.

Reader, S.M., Biro, D. 2010. Experimental identification of social learning in wild animals. Learning \& Behavior. 38, 265-283.

Sanz, C,, Morgan, D., Gulick, S., 2004. New insights into chimpanzees, tools, and termites from the Congo Basin. Am. Nat. 164, 567-581.

Sanz, C.M., Morgan, D.B., 2007. Chimpanzee tool technology in the Goualougo 
Triangle, Republic of Congo. J. Hum. Evol. 52, 420-433.

Sanz, C.M., Schöning, C., Morgan, D.B., 2009. Chimpanzees prey on army ants with specialized tool set. Am. J. Primatol. 71, 1-8.

4 Sanz and Morgan 2010

5 Schick, K. D. \& Toth, N. 1993. Making Silent Stones Speak. Simon and Schuster, New York.

7 Schick, K. D., Toth, N., Garufi, G., Savage-Rumbaugh, S., Rumbaugh, D.,

34 Tomasello, M., 1994. The question of chimpanzee culture. In: Wrangham, R.W., 
McGrew, W.C., de Waal, F.B.M., Heltne, P.G., Marquardt, L.A., (Eds),

Chimpanzee Cultures. Harvard University Press, Cambridge, Massachusetts \& London, England, pp. 301-318.

Tomasello, M., 1999. The Cultural Origins of Human Cognition. Harvard University Press, Cambridge, MA.

6 Tomasello 2009

7 Toth and Schick 2009

8 Uomini N, T., 2009. The prehistory of handedness: Archeological data and comparative ethology. J. Hum. Evol. 57, 411-419.

van Noordwijk, M.A., Sauren, S.E.B., Nuzuar Abulami, A., Morrogh-Bernard, H., Utami Atmoko, S.S., van Schaik, C.P., 2009. Development of independence. In: Wich, S.A., Mitra Setia, T., Utami Atmoko, S.A., van Schaik, C.P. (Eds), Orangutans Compared: Geographic Variation in Behavioral Ecology and Conservation Oxford University Press, Oxford, pp. 189-203.

van Schaik, C.P., 2004. Among Orangutans: Red Apes and the Rise of Human Culture. Harvard University Press, Cambridge, MA.

van Schaik, C. P. 2009. Geographic variation in the behavior of wild great apes: is it really cultural? In: Laland, K.N., Galef, B.G. (Eds), The Question of Animal Culture, Harvard University Press, Cambridge, pp. 70-98.

van Schaik, C.P., Deaner, R.O., Merrill, M.Y.,1999. The conditions for tool use in primates: implications for the evolution of material culture. J. Hum. Evol. 36, 719741.

van Schaik, C.P., Knott, C., 2001. Geographic variation in tool use on Neesia fruits in orangutans. Am J. Phys. Anthropol. 114, 331-342.

van Schaik, C.P., Pradhan, G.R., 2003. A model for tool-use traditions in primates: implications for the coevolution of culture and cognition, J. Hum. Evol. 44, 645-664. van Schaik, C.P., Ancrenaz, M., Borgen, G., Galdikas, B., Knott, C.D., Singleton, I., Suzuki, A., Utami-Atmoko, S.S., Merrill, M.Y., 2003a. Orangutan cultures and the evolution of material culture. Science 299, 102-105.

van Schaik, C.P., Fox, E.A., Fechtman, L.T., 2003b. Individual variation in the rate of use of tree-hole tools among wild orang-utans: implications for hominin evolution. J. Hum. Evol. 44, 11-23.

van Schaik, C. P. \& Burkart, J. M. 2010. Mind the gap: Cooperative breeding and the evolution of our unique features. In: Kappeler, P.M., Silk, J. (Eds), Mind the Gap: 
Tracing the origins of Human Universals. Springer, Berlin, pp. 477-496.

van Schaik, C.P., Burkart, J.M., 2011. Social learning and evolution: the cultural intelligence hypothesis. Phil. Trans. Roy. Soc., B. 366, 1008-1016.

Wich, S.A., Utami-Atmoko, S.S., Mitra Setia, T., Rijksen, H.D., Schurmann, C., van Hooff, J.A.R.A.M., van Schaik, C.P., 2004. Life history of wild Sumatran orangutans (Pongo abelii). J. Hum. Evol. 47, 385-398.

Whiten, A., Goodall, J., McGrew, W.C., Nishida, T., Reynolds, V., Sugiyama, Y., Tutin, C.E.G., Wrangham, R.W., Boesch, C., 1999. Cultures in chimpanzees. Nature 399, 682-685.

Whiten, A., van Schaik, C.P., 2007. The evolution of animal 'cultures' and social intelligence. Phil. Trans. Roy Soc., B. 362, 603-620.

Whiten, A., Spiteri, A., Horner, V., Bonnie, K.E., Schapiro, S.J., de Waal, F.B.M., 2007. Transmission of multiple traditions within and between chimpanzee groups. Curr. Biol. 17, 1-6.

Whiten, A., Mesoudi, A., 2008. Establishing an experimental science of culture: animal social diffusion experiments. Phil. Trans. Roy. Soc., B. 363, 3477-3488.

Whiten, A., McGuigan, N., Marshall-Pescini, S., Hopper, L., 2009. Emulation, imitation, over-imitation and the scope of culture for child and chimpanzee. Phil. Trans. Roy. Soc., B. 364, 2417-2428.

Wyles, J.S., Kunkel, J.G., Wilson, A.C., 1983. Birds, behavior, and anatomical evolution. Proc. Natl. Acad. Sci., USA. 80, 4394-4397.

Wynn, T., Hernandez-Aguilar, R.A., Marchant, L.F., McGrew, W.C., 2011. "An ape's view of the Oldowan" revisited. Evol. Anthropol, 20:181-197.

Yamamoto, S., Yamakoshi, G., Humle, T., Matsuzawa, T., 2008. Invention and modification of a new tool use behavior: Ant-fishing in trees by a wild chimpanzee (Pan troglodytes virus) at Bossou, Guinea. Am. J. Primatol. 70, 699-702.

Zacks, J. M., Tversky, B. 2001. Event structure in perception and conception. Psychological Bulletin. 127, 3-21. 


\section{Figure captions}

2 Figure 1: Flow chart showing the details of the simulation.

3 Final results are obtained by averaging over 200 such realizations.

5 Figure 2: Examples of scenarios in which technology at various levels evolved, showing

6 the impact of sociability in comparison with the innovative ability in hypothetical great

7 ape populations.

8 The plots show the equilibrium proportion of population using tools at technology levels 1

9 through 3 (TL1-3) as a function of time, assuming that TL3 is the highest attainable

10 complexity of tools. (a) the parameter values representative of a hypothetical, typical

11 great ape population $(N=501, \alpha=0.2, \kappa=1, \varepsilon=0.0001, \mu=0.05, \lambda=15$; see Table

122 for definitions). Only TL1 can establish itself.

13 (b) If sociability is increased to $\kappa=2$, keeping rest of the parameters same as in (a), the

14 majority of the population is found to reach TL3.

15 (c) If the sociability is still at the typical great ape level, but innovative ability $\varepsilon$ is

16 increased by two orders of magnitude $(\varepsilon=0.01)$ compared to that in (a), only TL1 and

17 TL2 are common, whereas only a small proportion reaches to TL3.

19 Figure 3: Variation in proportion of population using tools of varying complexity (TL1-3)

20 as a function of sociability, $\kappa$. The error bars represent the standard deviation in the

21 simulation outcomes.

22 The rest of the parameter values are as in Figure 2, viz., $N=501, \alpha=0.2, \varepsilon=0.0001$,

$23 \mu=0.05, \lambda=15$; see Table 2 for definitions. For a fixed low social-learning ability, $\alpha$, as

$24 \kappa$ varies from representative values for great apes to early humans, the major changes

25 in the proportion of the population with tools at TL3 occur as $\kappa$ increases from $\kappa=1$

26 through $\kappa=3$ (i.e. from 2 to 6 tolerant experts).

28 Figure 4: Proportion of the population using tools of varying complexity (TL1-3) as a

29 function of time for varying population size.

30 Graphs show the proportion of the population using tools of technology level TL1 (a),

31 TL2 (b), or TL3 (c), for various values of $N$ assuming that TL3 is the highest attainable

32 complexity of tools. The rest of the parameters are: $\alpha=0.2, \kappa=2, \varepsilon=0.0001$, 
$1 \mu=0.05, \lambda=15$; see Table 2 for definitions. The total population size $N$ affects only the

2 time needed to reach equilibrium but not the equilibrium value.

3

4 Figure 5: Proportion of the population using TL3 tools as a function of social learning

5 ability, $\alpha$.

6 Two curves correspond to $\kappa=1$ (solid curve; corresponds to low sociability) and

$7 \quad \kappa=3$ (dotted curve; corresponds to higher sociability), respectively. The rest of the

8 parameters are set to: $N=501, \varepsilon=0.0001, \mu=0.05, \lambda=15$; see Table 2 for

9 definitions. An increase of $\alpha=0.2$ to $\alpha=0.3$ provides a strong boost to accumulation of

10 technology, even at low $\kappa$ (solid curve). Similarly high levels could be reached at even

11 lower a values for higher sociability (dotted curve).

12

13 Figure 6: Proportion of the population using tools of varying complexity as a function of

14 time for varying innovation rates: (a) TL1, (b) TL2, and (c) TL3.

15 The inventive ability, $\varepsilon$, is varied assuming that TL3 is the highest attainable complexity

16 of tools. The rest of the parameters are: $\alpha=0.4$ (higher than great ape level), $\kappa=2$,

$17 \mu=0.05, \lambda=15$; see Table 2 for definitions. Even orders of magnitude change in $\varepsilon$ only

18 affects the time needed to reach equilibrium but not the equilibrium value.

19

20 Figure 7: The culture pyramid (modified after Whiten and van Schaik, 2007), showing the

21 hierarchical or nested-subset nature of various manifestations of culture.

22 
Table 1.

Definitions and examples of technology levels. As one moves down in the table, technological accumulation increases. It is assumed that each level has reached high prevalence through cultural transmission. References are given only for cases not mentioned in the description of the model.

\begin{tabular}{|c|c|c|c|}
\hline $\begin{array}{l}\text { Techno- } \\
\text { logy level }\end{array}$ & Description & Examples sticks & Examples stones \\
\hline TLO & $\begin{array}{l}\text { A single action (use } \\
\text { object as tool) }\end{array}$ & $\begin{array}{l}\text { Use a stick found } \\
\text { nearby to poke into hole }\end{array}$ & $\begin{array}{l}\text { Use a stone found nearby } \\
\text { to pound nuts or bones }{ }^{a}\end{array}$ \\
\hline TL1 & $\begin{array}{l}\text { A single action, } \\
\text { followed by other } \\
\text { coordinated action } \\
\text { (use object as tool on } \\
\text { prepared substrate) }\end{array}$ & $\begin{array}{l}\text { Take a stout branch } \\
\text { found elsewhere to a } \\
\text { suitable anvil and use } \\
\text { as a wooden hammer }\end{array}$ & $\begin{array}{l}\text { Take a stone found } \\
\text { elsewhere to a suitable } \\
\text { anvil, and use to pound }\end{array}$ \\
\hline TL1 & $\begin{array}{l}\text { A single action or set } \\
\text { of closely related } \\
\text { actions on one object, } \\
\text { which is subsequently } \\
\text { used as a tool (tool } \\
\text { manufacture) }\end{array}$ & $\begin{array}{l}\text { Break a twig from a } \\
\text { branch, trim to size (and } \\
\text { perhaps remove side- } \\
\text { twigs, etc.), and use as } \\
\text { tool }\end{array}$ & $\begin{array}{l}\text { Hit stone on hard surface } \\
\text { to produce flakes, through } \\
\text { the anvil or throwing } \\
\text { techniques }{ }^{\text {b }} \text {, and use flakes } \\
\text { as tool }\end{array}$ \\
\hline TL2 & \begin{tabular}{|l|} 
Two distinct, \\
subsequent actions on \\
one object, which is \\
subsequently used as \\
tool (composite tool)
\end{tabular} & $\begin{array}{l}\text { Prepare a twig to } \\
\text { become a probe, and } \\
\text { subsequently fray the } \\
\text { end of the probe, thus } \\
\text { improving its efficiency }\end{array}$ & $\begin{array}{l}\text { Not applicable due to body } \\
\text { restrictions (body actions } \\
\text { on stones are } \\
\text { meaningless) }\end{array}$ \\
\hline TL2 & \begin{tabular}{|l|} 
Integrated actions on \\
two distinct objects, \\
which are each \\
produced separately \\
(tool set)
\end{tabular} & $\begin{array}{l}\text { Use of a separately } \\
\text { prepared perforating } \\
\text { stick to create a tunnel, } \\
\text { followed by use of } \\
\text { separately produced } \\
\text { probe to extract } \\
\text { termites }^{c}\end{array}$ & $\begin{array}{l}\text { Not applicable due to body } \\
\text { restrictions }\end{array}$ \\
\hline TL2 & \begin{tabular}{|l|} 
Co-action, two \\
carefully integrated \\
actions on two objects, \\
one in each hand
\end{tabular} & $\begin{array}{l}\text { Not applicable due to } \\
\text { material restrictions } \\
\text { (wood vs. stones) }\end{array}$ & $\begin{array}{l}\text { Hitting a hand-held stone } \\
\text { core with a stone hammer } \\
\text { to produce an Oldowan } \\
\text { flake (using hard-hammer } \\
\text { percussion or bipolar } \\
\text { technique) b }\end{array}$ \\
\hline TL3 & $\begin{array}{l}\text { Use a made tool to } \\
\text { modify another tool } \\
\text { (combining } \\
\text { manufactured tools) }\end{array}$ & $\begin{array}{l}\text { Not applicable due to } \\
\text { material restrictions }\end{array}$ & $\begin{array}{l}\text { Use an Oldowan flake, } \\
\text { produced earlier, to } \\
\text { sharpen a stick for more } \\
\text { effective use }\end{array}$ \\
\hline TL3 & \begin{tabular}{|l|} 
Use co-action (TL2) \\
many times in a \\
coordinated sequence
\end{tabular} & $\begin{array}{l}\text { Not applicable due to } \\
\text { material restrictions }\end{array}$ & $\begin{array}{l}\text { Produce an Acheulean } \\
\text { hand axe }\end{array}$ \\
\hline
\end{tabular}




\begin{tabular}{|l|l|l|l|}
\hline$T L 3$ & $\begin{array}{l}\text { Use two different co- } \\
\text { actions in integrated } \\
\text { sequence }\end{array}$ & $\begin{array}{l}\text { Not applicable due to } \\
\text { material restrictions }\end{array}$ & $\begin{array}{l}\text { Use hard hammer to } \\
\text { prepare a core, followed by } \\
\text { soft hammer, to produce } \\
\text { flakes off an Acheulean } \\
\text { handaxe }\end{array}$ \\
\hline TL3 & $\begin{array}{l}\text { Integrated actions on 3 } \\
\text { distinct objects, which } \\
\text { are each produced } \\
\text { separately }\end{array}$ & $\begin{array}{l}\text { Use of separately } \\
\text { prepared pounding } \\
\text { stick, followed by a lever } \\
\text { tool, followed by a dip } \\
\text { stick to obtain honey } \\
\text { from bee nests }\end{array}$ & $\begin{array}{l}\text { Not applicable due to body } \\
\text { restrictions }\end{array}$ \\
\hline TL4 & $\begin{array}{l}\text { As in TL3 above, then } \\
\text { add resharpening with } \\
\text { different hammer }\end{array}$ & $\begin{array}{l}\text { Not applicable due to } \\
\text { material restrictions }\end{array}$ & $\begin{array}{l}\text { As in TL3 above, followed } \\
\text { by resharpening }\end{array}$ \\
\hline
\end{tabular}

a- as in monkeys: Gumert et al., 2009; Ottoni and Izar, 2008; b- Schick and Toth, 1993; Boysen, personal communication; c- Sanz et al., 2009; d- Sanz and Morgan, 2007; eDominguez-Rodrigo et al., 2001. 


\section{Table 2.}

2 Definition of the main parameters used in the model and the best estimates of the same

3 for great apes (details of the estimates are provided in the SI).

\begin{tabular}{|c|c|c|}
\hline Parameter & Description & $\begin{array}{l}\text { Best estimates for great } \\
\text { apes (details in SI) }\end{array}$ \\
\hline$\varepsilon(0 \leq \varepsilon \leq 1)$ & $\begin{array}{l}\text { Inventive ability: Probability of } \\
\text { acquiring a particular skill in one time } \\
\text { step in the absence of social } \\
\text { influence }\end{array}$ & $\begin{array}{l}0.0001 \text { (i.e. approximately one } \\
\text { in } 500 \text { individuals invents } \\
\text { assuming annual mortality rate } \\
\text { at } 5 \% \text { ) }\end{array}$ \\
\hline$\alpha(0 \leq \alpha \leq 1)$ & $\begin{array}{l}\text { Social learning ability: Probability of } \\
\text { learning a particular skill in one time } \\
\text { step under social influence from any } \\
\text { of the skilled neighbors }\end{array}$ & $\begin{array}{l}0.2 \text { (rather low value because } \\
\text { great apes are not good } \\
\text { copiers) }\end{array}$ \\
\hline$\kappa(1 \leq 2 \kappa \leq N)$ & $\begin{array}{l}\text { Sociability, or opportunities for social } \\
\text { learning: Number of individuals in the } \\
\text { social unit that are directly connected } \\
\text { to the focal individual as possible } \\
\text { experts from whom social learning is } \\
\text { possible. The focal individual has } 2 \mid \\
\text { nearest neighbors to learn from. The } \\
\text { parameter | is a constant for a given } \\
\text { regular graph (the number of } \\
\text { connected neighbors on one side of } \\
\text { the individual). }\end{array}$ & $\begin{array}{l}1 \text { (i.e. association with only two } \\
\text { individuals most of the time: } \\
\text { mother and another associate) }\end{array}$ \\
\hline$\mu$ & $\begin{array}{l}\text { Annual mortality: proportion dying } \\
\text { each year }\end{array}$ & $\begin{array}{l}0.05 \text { (average rate if orangutan } \\
\text { and chimpanzee populations } \\
\text { considered together) }\end{array}$ \\
\hline$\lambda$ & $\begin{array}{l}\text { Age marking the end of active social } \\
\text { learning ends }\end{array}$ & About 15 years \\
\hline$M$ & Maximum age reached in the wild & 50 years \\
\hline$N$ & Population size & About 500 \\
\hline
\end{tabular}




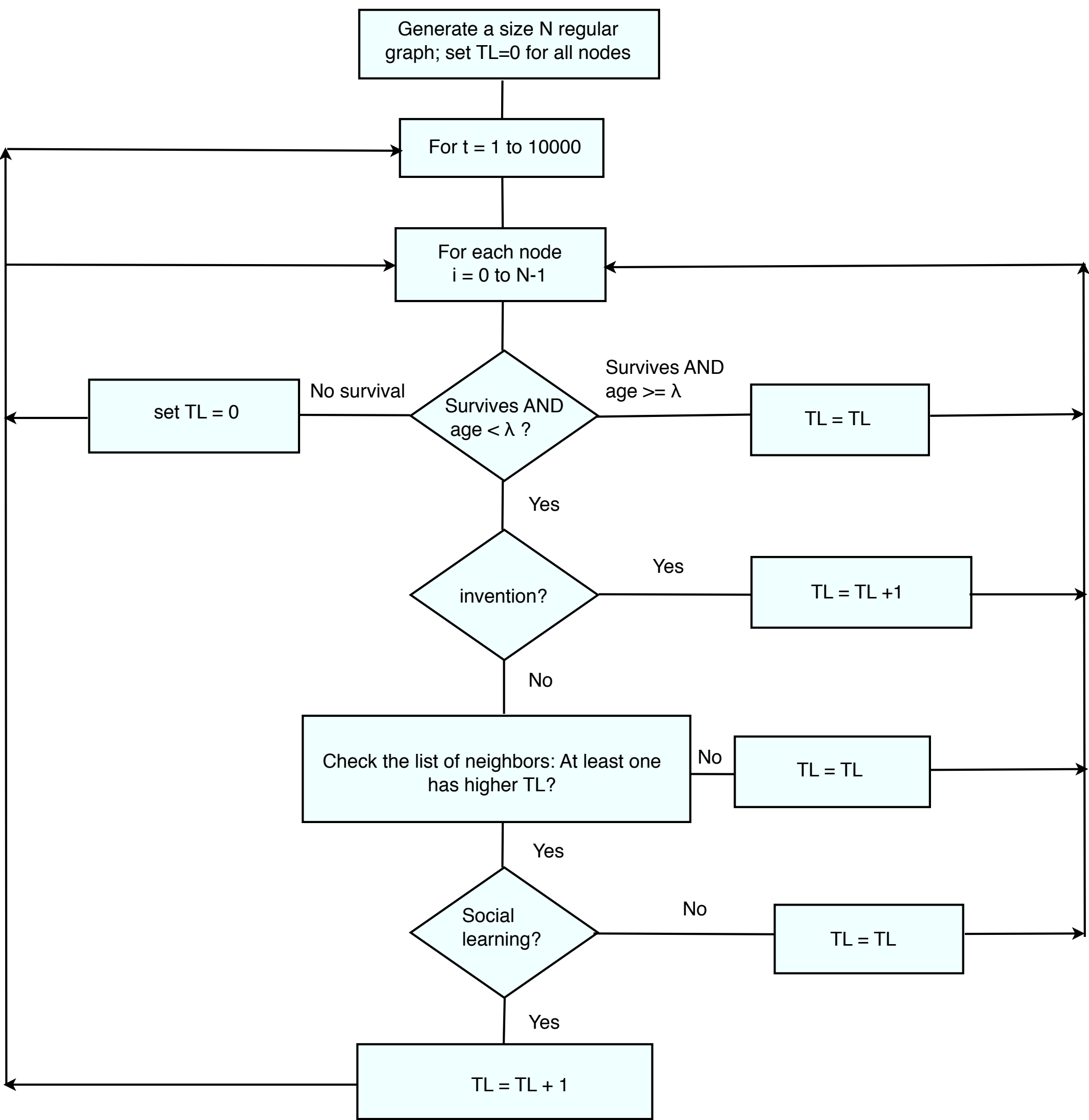




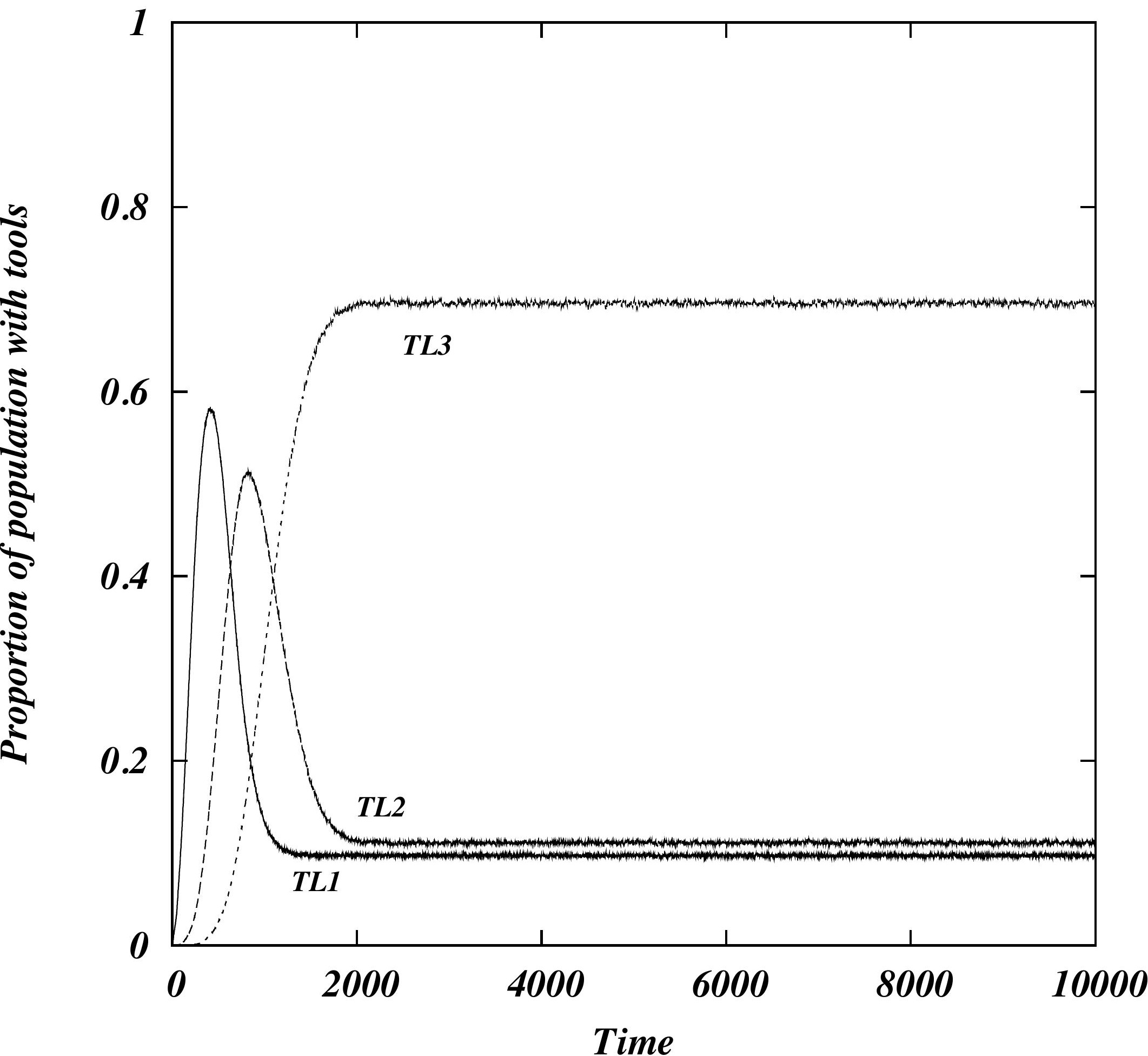


Figure 3

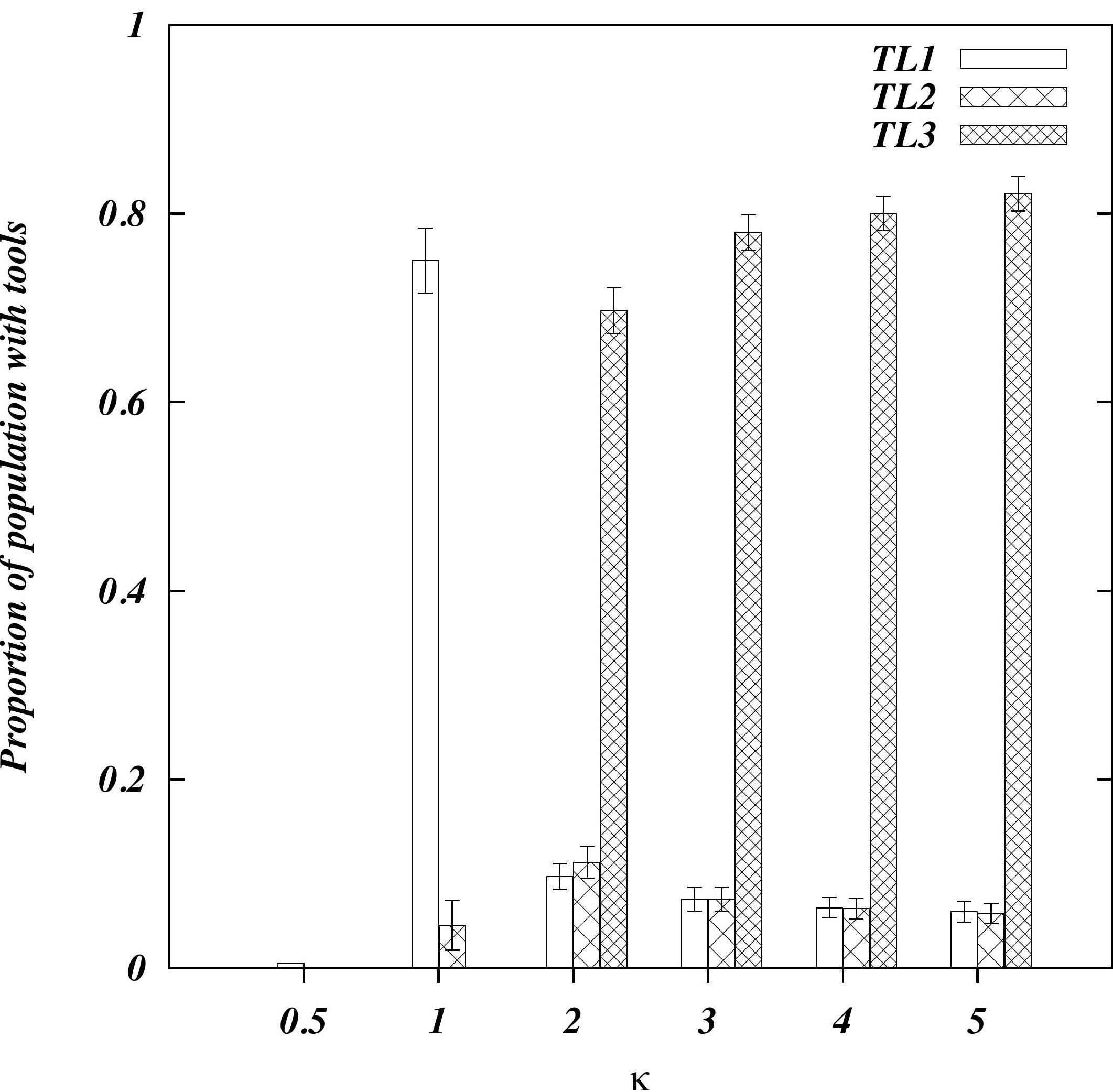


Figure 4

(a) TLI

0.8
0.6

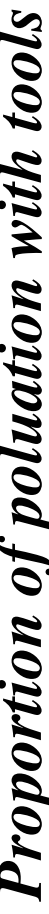

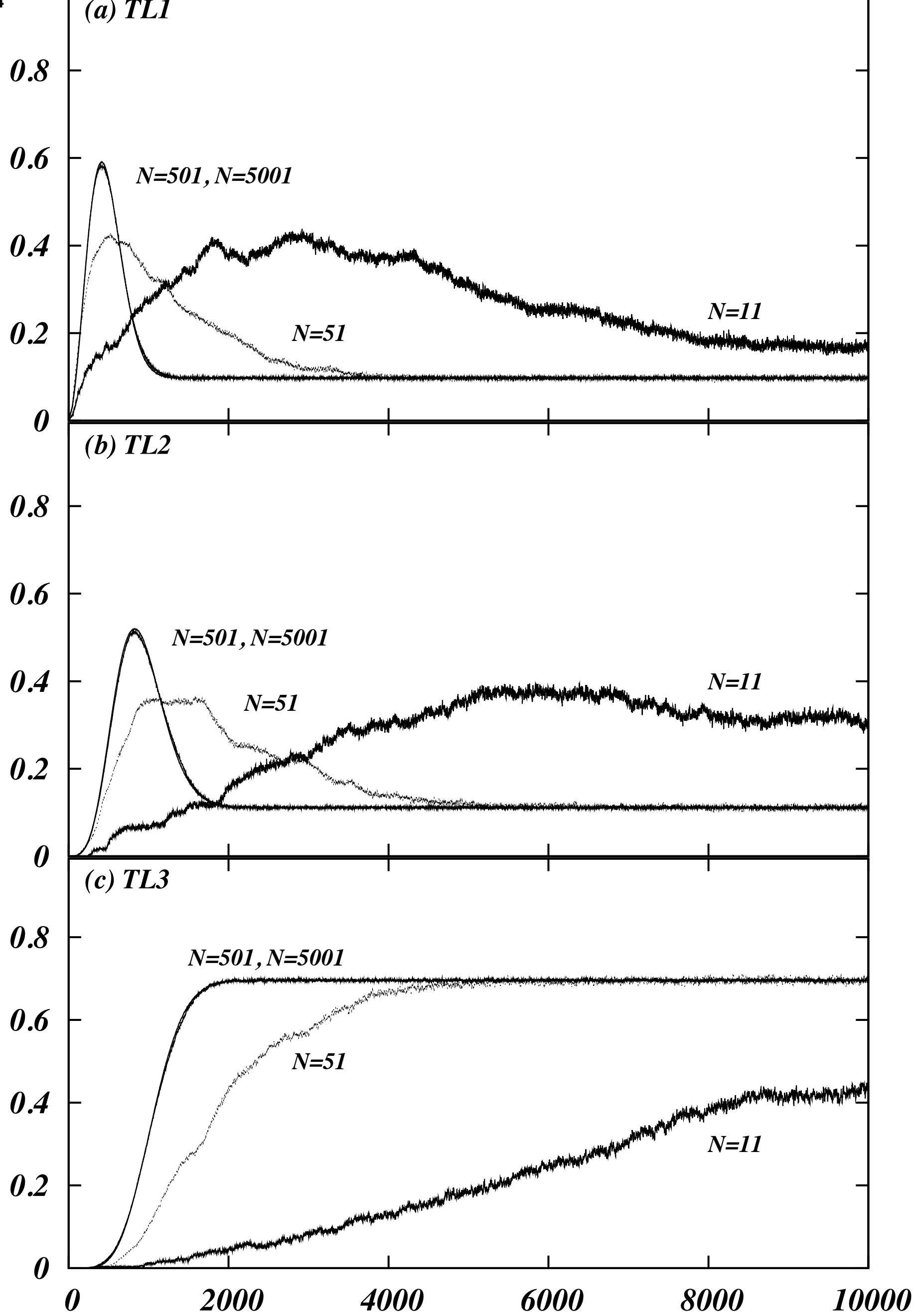

Time 


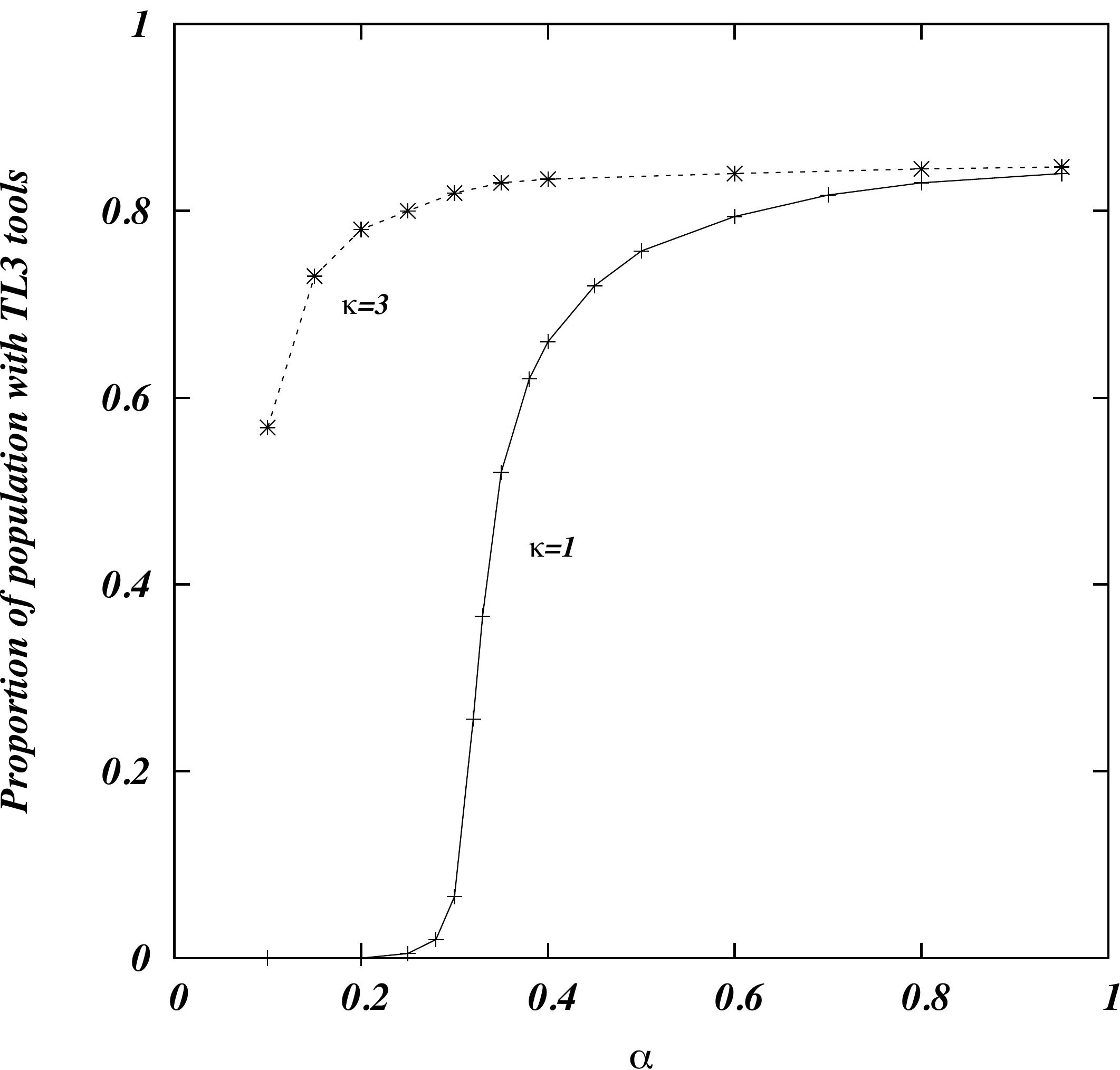


Figure 6

(a) TLI

0.8

0.6

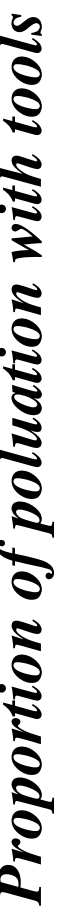

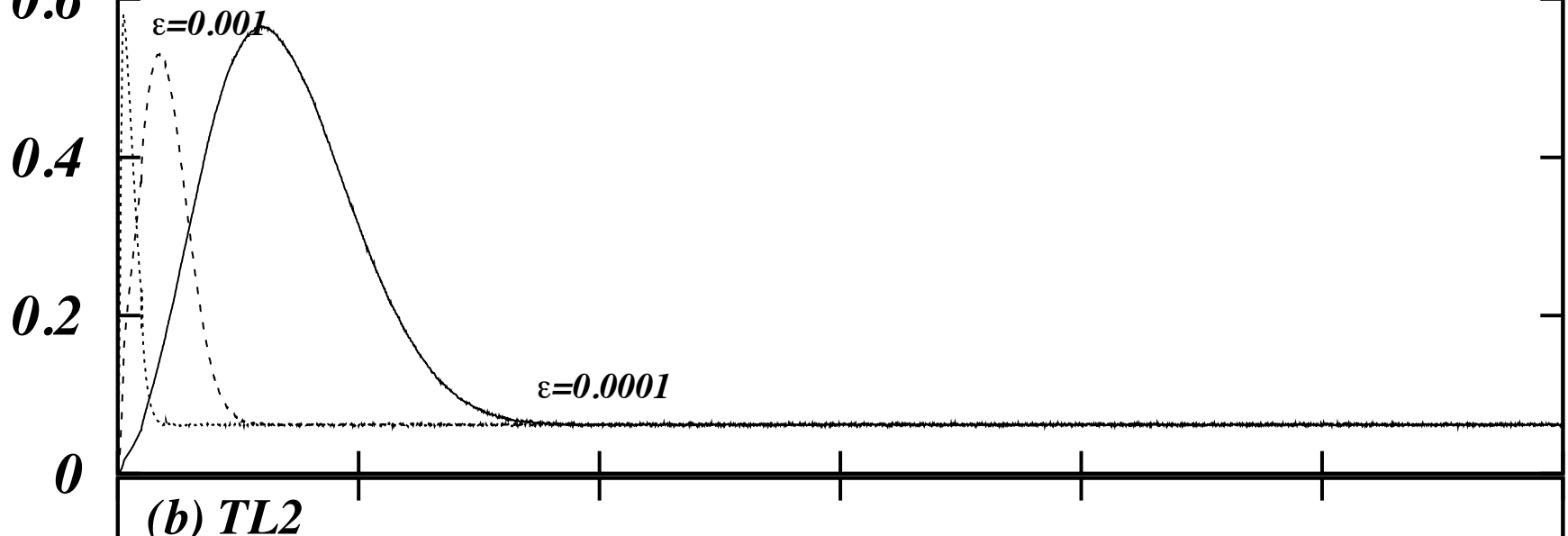

(b) $T L 2$

0.8
0.6

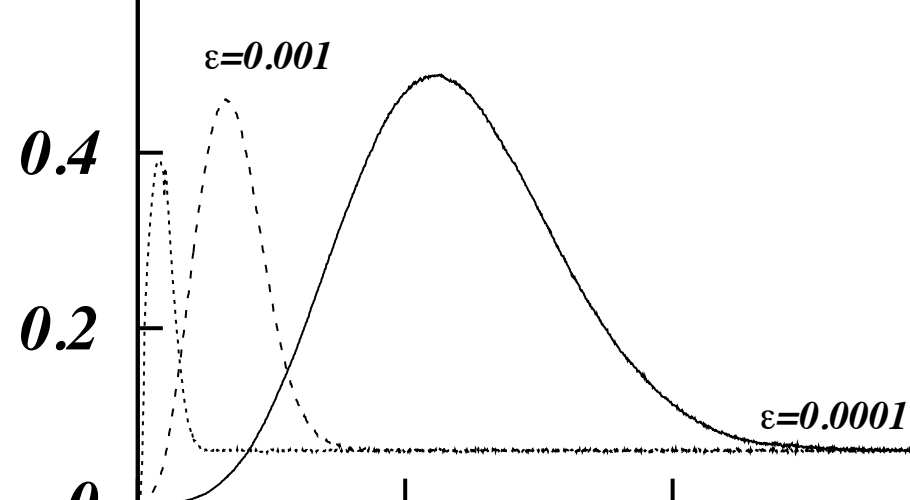

(c) $\mathrm{TL3}$

$0.8-\varepsilon=0.01$

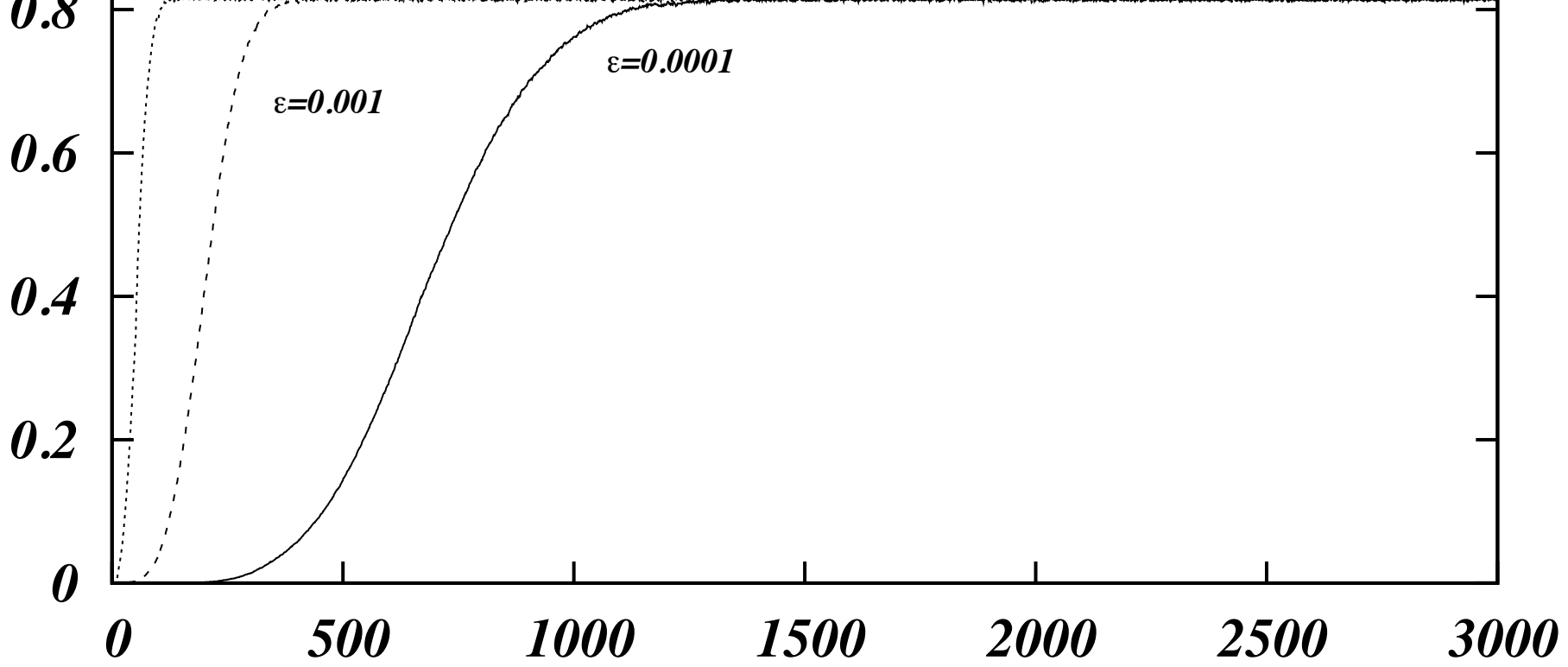

Time 


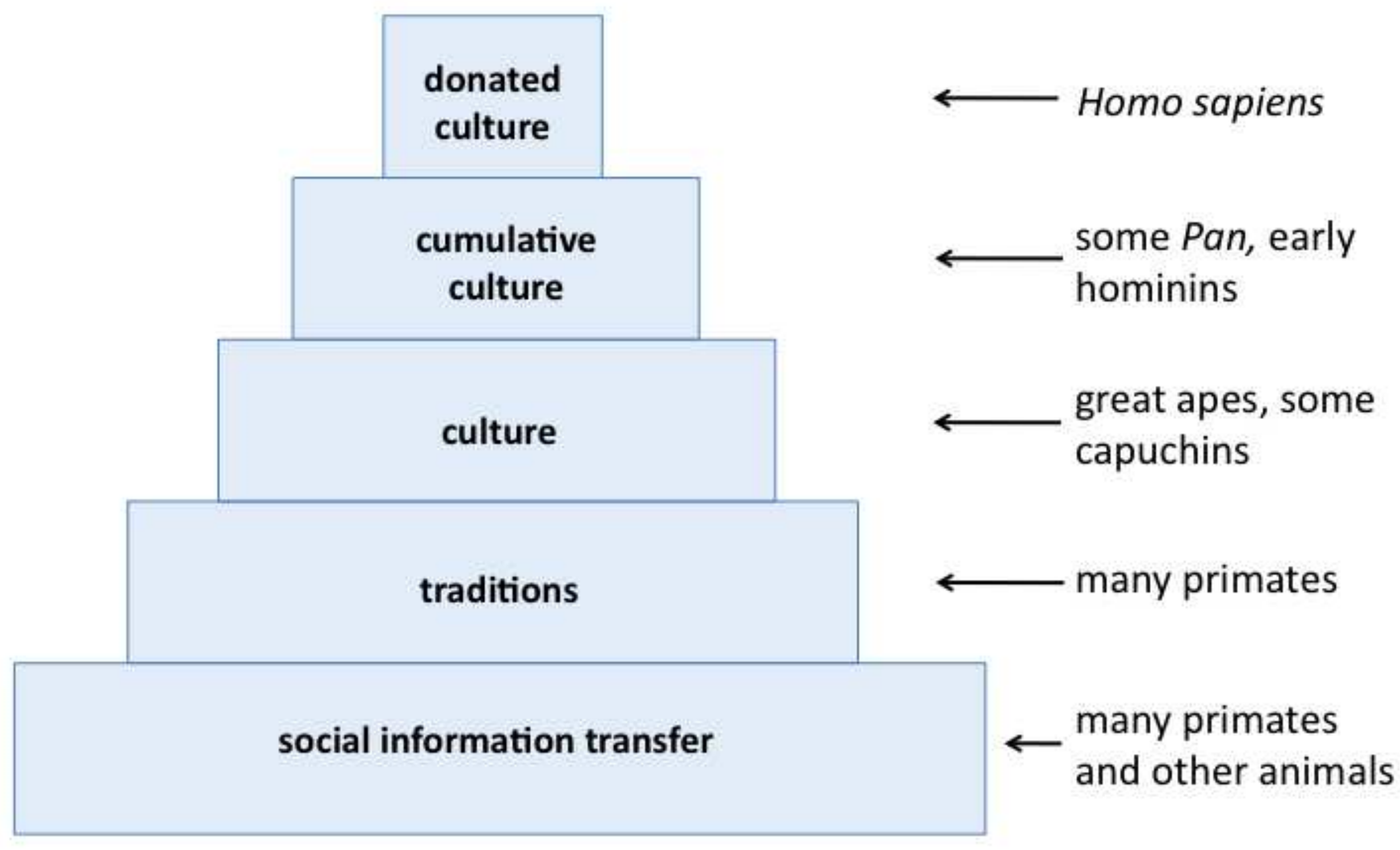

